\title{
Construction of Wind Turbine Bearing Vibration Monitoring and Performance Assessment System
}

\author{
Feng-Tai $\mathrm{Wu}^{1 *}$, Chun-Chieh Wang ${ }^{1}$, Jui-Hung Liu ${ }^{2}$, Chia-Ming Chang ${ }^{2}$, Ya-Ping Lee ${ }^{1}$ \\ ${ }^{1}$ Mechanical and Systems Research Laboratories, Industrial Technology Research Institute, Hsinchu, Taiwan; ${ }^{2}$ Green Energy and \\ Environment Research Laboratories, Industrial Technology Research Institute, Hsinchu, Taiwan. \\ Email: *TerryWu@itri.org.tw
}

Received September $23^{\text {rd }}, 2013$; revised October $21^{\text {st }}, 2013$; accepted October $27^{\text {th }}, 2013$

Copyright (C) 2013 Feng-Tai Wu et al. This is an open access article distributed under the Creative Commons Attribution License, which permits unrestricted use, distribution, and reproduction in any medium, provided the original work is properly cited.

\begin{abstract}
This study is primary to develop relevant techniques for the bearing of wind turbine, such as the intelligent monitoring system, the performance assessment, future trend prediction and possible fault classification etc. The main technique of system monitoring and diagnosis is divided into three algorithms, such as the performance assessment, performance prediction and fault diagnosis, respectively. Among them, the Logistic Regression (LR) is adopted to assess the bearing performance condition, the Autoregressive Moving Average (ARMA) is adopted to predict the future variation trend of bearing, and the Support Vector Machine (SVM) is adopted to classify and diagnose the possible fault of bearing. Through testing, this intelligent monitoring system can achieve real-time vibration monitoring, current performance assessment, future performance trend prediction and possible fault classification for the bearing of wind turbine. The monitor and analysis data and knowledge not only can be used as the basis of predictive maintenance, but also can be stored in the database for follow-up off-line analysis and used as the reference for improvement of operation parameter and wind turbine system design.
\end{abstract}

Keywords: Signal Processing; Feature Extraction; Performance Assessment; Performance Prediction; Fault Diagnosis

\section{Introduction}

At present, the comparatively attractive wind power generator can be mainly divided into onshore wind turbine and offshore wind turbine in accordance with the setup location. The maintenance time of wind turbine can be divided into the predictable periodic maintenance and the unpredictable breakdown repair. When the unpredictable breakdown is occurred, such as the breakdown of gearbox, several months may be required for the repair, lifting, or waiting for material, which may cause large loss of windfarm. In order to prevent the unpredictable breakdown, the intelligent monitoring and diagnosis analytical technique can be used to assure the normal condition of whole wind turbine, and the preventive maintenance schedule can be arranged for key components [1-3].

The base for intelligent monitoring and diagnosis analytical technique of wind power generator system is the setup of Condition Monitoring System (CMS). The purpose of CMS is to monitor the operation condition of key

\footnotetext{
${ }^{*}$ Corresponding author.
}

parts in each subsystem of wind turbine, such as the vibration for the parts of transmission system and generator. The physical signals are analyzed statistically through breakdown diagnosis, in order to assess the condition and probable failure type of monitoring subject. Finally, the alarm is provided in accordance with the analytical conclusion to prevent unpredictable breakdown and major failure, in order to raise the usability and reliability of whole wind turbine system. The CMS can grasp the condition of wind turbine system to reduce the unpredictable maintenance cost effectively, especially to the offshore wind turbine system. Because the maintenance of offshore wind turbine system must be matched with the schedule of fleet, and the uncertainty of weather shall also be considered, so when the unpredictable breakdown is occurred, extra maintenance expenses, schedule delay and substitute power generation will cause large loss of relevant firm. Through the advanced alarm of intelligent maintenance system, not only the unpredictable breakdown can be reduced, but also the arrangement and deployment of operation and maintenance schedule can be 
conducted, in order to reach the requirements of fastest maintenance achievement and lowest maintenance cost by matching the maintenance facility and the manpower deployment [4-7].

Figure 1 shows the annual failure rate and downtime per failure for every subsystem in accordance with LWK and WMEP wind turbine statistical database specified in [5], wherein the statistical data for the operation status of about 20,000 wind turbines operated in 13 years are adopted. As shown in Figure 1, it is observed that there is higher failure rate for the electric system and electric control. Although the failure rate is higher for these electric devices, the replacement cost is not high, the spare parts are easy to be obtained, and the repair time of failure is pretty short, then the influence on downtime of whole wind turbine system is not significant. On the contrary, the failure rate of large-scale devices such as gearbox, drive train, generator, rotor blades is very low, less than one time per year, but once these devices break down, if there are no spare parts ready in advance, the wind turbine must shut down and wait for the material, which will elongate the downtime. Figure 2 shows the comparison for the failure location of electric machine in wind turbine. It is found that there is very high failure rate at the part of bearing. So the condition monitoring and fault diagnosis techniques have become very important and been the center of attention [1-7].

The maintenance way of mechanical equipment can be divided into the breakdown maintenance, time-based maintenance, preventive maintenance, and predictive maintenance in accordance with the maintenance timing. Figure 3 shows the difference for comparison of periodic maintenance and predictive maintenance. If the CMS is added in relevant equipment of wind turbine, the maintenance period can be lengthened greatly compared to traditional periodic maintenance. Not only many unnecessary maintenance times can be reduced, but the unpredictable breakdown due to severe damage of components can also be avoided. So, if the optimal predictive maintenance can be arranged in accordance with the monitor-

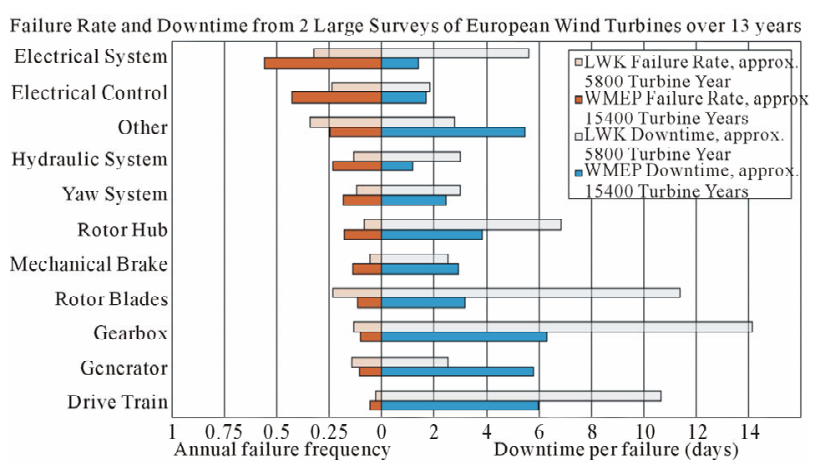

Figure 1. Annual failure rate and downtime per failure for every subsystem of wind turbine [5].

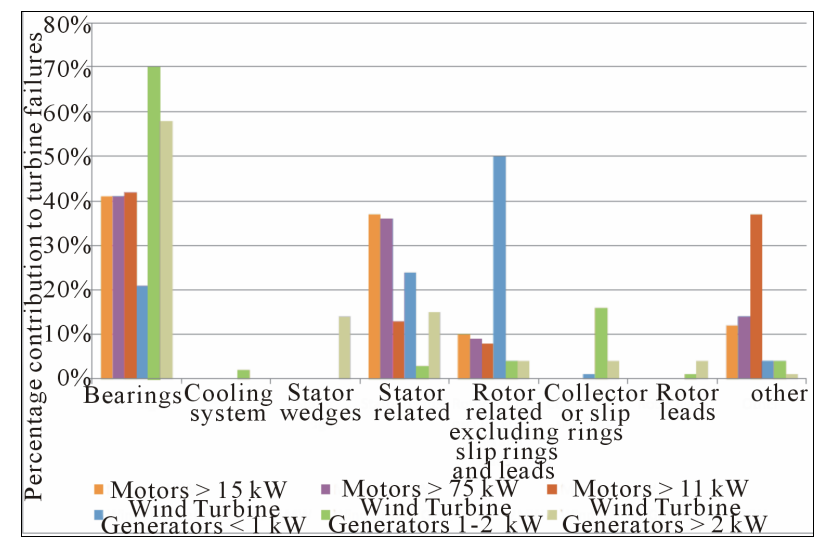

Figure 2. Ratio for failure location of electric machine in wind turbine [5].

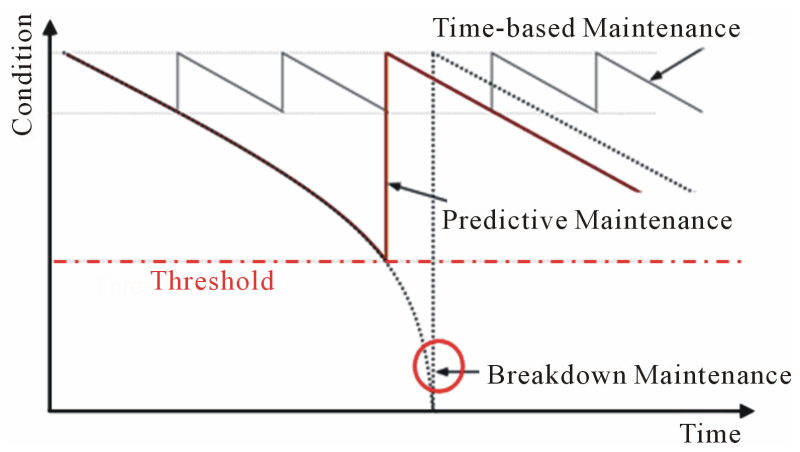

Figure 3. Difference for comparison of periodic maintenance and predictive maintenance [8-10].

ing and diagnosing condition, not only the downtime can be reduced, but the optimal maintenance schedule can also be planned, in order to shorten the failure time [8-10].

\section{Intelligent Monitoring System Structure of Wind Turbine}

The goal of intelligent monitoring system developed by this study is to integrate the information and communication technique and equipment condition monitoring technique, and apply it to the intelligent maintenance for key components of wind turbine. This intelligent system is featured in the intelligent, determined and web-based real-time monitoring and detecting function, which can effectively raise the operation efficiency and maintenance service ability of wind turbine.

The intelligent monitoring system of wind turbine mainly provides the monitoring, predicting and preventing services, in order to avoid unpredictable breakdown of wind turbine, and raises the usability of wind turbine through predictive maintenance mechanism to maintain stable power generation ability of wind turbine. The operation mechanism of this system is to summarize relevant operation data through the parameter collection de- 
vice of wind turbine first, and transmit these data to monitoring database of cloud computing platform through internet. Then, these data are analyzed, assessed, and predicted by the computing modules of cloud computing platform, and the analysis data are displayed by graph on the operation interface of client end through internet. If there is any abnormal condition, relevant operation personnel will be noticed through message or email, in order to achieve the purpose of quickest troubleshooting.

The simple structure for intelligent monitoring system of wind turbine is shown in Figure 4, which mainly includes the cloud computing platform (including Webbased server and application server) and Data Acquisition (DAQ) equipment. The user can operate the system by the mobile device, notebook or personal computer through internet. In addition, the system can provide the alarm function for the user to deal with abnormal condition of wind turbine in time. The user can also use realtime graph to display the analysis and comparison data of cloud computing, in order to grasp the performance condition and relevant index of wind turbine totally. The monitoring hardware setup of system is shown in Figure 5. An anemometer, a tachometer and a triaxial accelerometer are installed at the top of tower. Two seismic accelerometers are installed at the 1st floor and 2nd floor of tower column, respectively. The triaxial accelerometer is used to measure the vibration signal of main bearing, and the seismic accelerometers are used to measure the vibration signal of tower column.

As shown in Figure 6, the monitoring system for performance assessment and diagnosis of wind turbine bearing is mainly composed of three computing modules. First, the LR algorithm is adopted to assess the bearing performance condition (confidence value, $\mathrm{CV}$ ). Then, the ARMA algorithm is adopted to predict the future performance variation trend of bearing in accordance with known bearing performance record. When possible fault

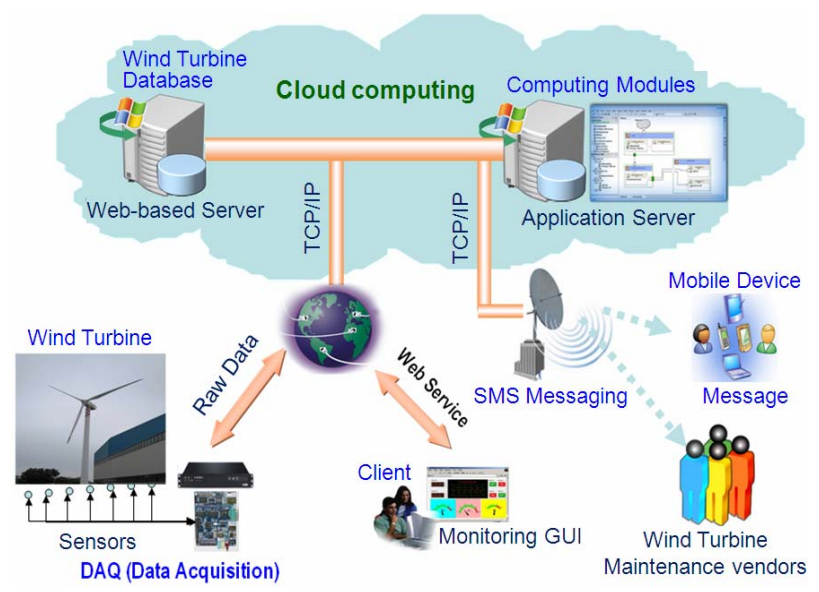

Figure 4. Structure for intelligent monitoring system of wind turbine.

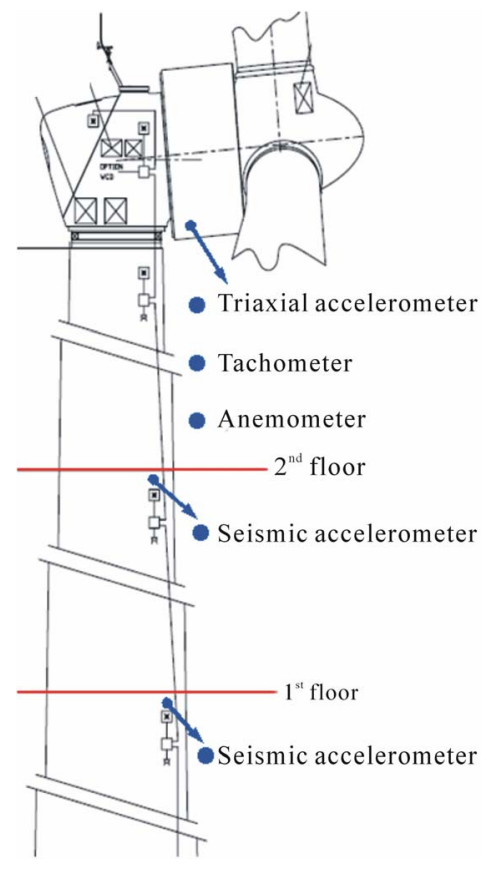

Figure 5. Monitoring hardware setup of wind turbine.

of bearing is occurred, the SVM algorithm is adopted to classify and diagnose the possible fault of bearing. The relevant technique for setting up intelligent monitoring system is relatively mature and there are many successful application examples, the required technique, such as assessment, diagnosis, prediction, classification and data mining can be found in [10-18]. The monitoring system developed by this study will select suitable and stable algorithms to conduct the development, integration, and test analysis. The rules and preliminary test results of the abovementioned three computing modules will be described as follows.

\section{Performance Assessment for Wind Turbine Bearing}

\subsection{LR Algorithm [19]}

The Logistic Regression (LR) algorithm is mainly used to assess dichotomous problems. If the performance assessment result of wind turbine lies between normal and abnormal condition, this algorithm may be adopted. The purpose of LR algorithm is to obtain the optimal model for representing output dependent variable $g(\mathbf{x})$ and input independent variable $\mathbf{x}$. Then, this model is represented by the logistic model to reveal the probability $P(\mathbf{x})$ of event represented by the output dependent variable. This probability is ranged between 0 and 1 . $P(\mathbf{x})$ is a logistic function, which is shown as Equation (1).

$$
P(\mathbf{x})=\frac{1}{1+\mathrm{e}^{-g(\mathbf{x})}}=\frac{\mathrm{e}^{g(\mathbf{x})}}{1+\mathrm{e}^{g(\mathbf{x})}} .
$$




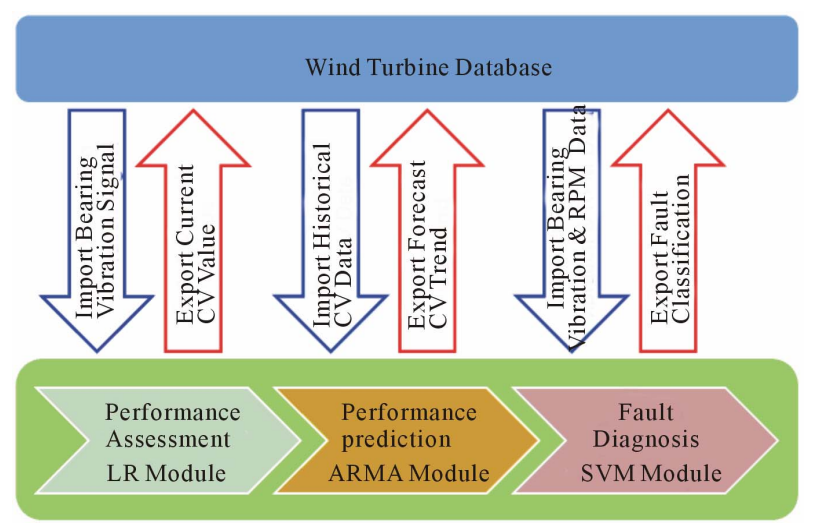

Figure 6. Module structure for performance assessment and diagnosis of wind turbine bearing.

The logistic model can be shown as Equation (2).

$$
\log \frac{P(\mathbf{x})}{1-P(\mathbf{x})}=\alpha+\beta_{1} x_{1}+\beta_{2} x_{2}+\cdots+\beta_{K} x_{K} .
$$

From Equation (2) it is known that the logistic model is $g(\mathbf{x})$, which is the linear combination of input independent variable $\mathbf{x}$ and its corresponding coefficient $\boldsymbol{\beta}$.

This study will adopt the abovementioned logistic function $P(\mathbf{x})$ as the Confidence Value $(\mathrm{CV})$ for assessing the performance index of wind turbine bearing. $\mathrm{CV}$ can be shown as Equation (3).

$$
C V(\mathbf{x})=\frac{1}{1+\mathrm{e}^{-\left(\alpha+\beta_{1} x_{1}+\beta_{2} x_{2}+\cdots+\beta_{K} x_{K}\right)}} .
$$

where the variables $x_{1}$ to $x_{K}$ represent the statistical feature of time domain and relevant bearing feature of frequency domain calculated in accordance with the bearing vibration signal. The corresponding coefficients $\alpha$ and $\beta_{1}$ to $\beta_{K}$ should be obtained from the training in advance. First, sufficient bearing vibration signals at normal condition are selected to calculate the representative feature group of time domain and frequency domain. The $C V$ of these feature groups is defined as 0.95 (95\%). Then, sufficient bearing vibration signals at abnormal condition are selected to calculate the representative feature group of time domain and frequency domain. Then the $C V$ of these feature groups is defined as 0.05 (5\%). The corresponding coefficients of logistic model are obtained from these known feature groups at normal condition and abnormal condition. After the corresponding coefficients $\alpha$ and $\beta_{1}$ to $\beta_{K}$ are obtained by the maximum likelihood estimator, the CV value of current bearing performance index can be calculated by Equation (3).

\subsection{Performance Assessment Test of Wind Turbine Bearing}

Figure 7 shows the monitoring database of wind turbines tested in this study, and the record period was from
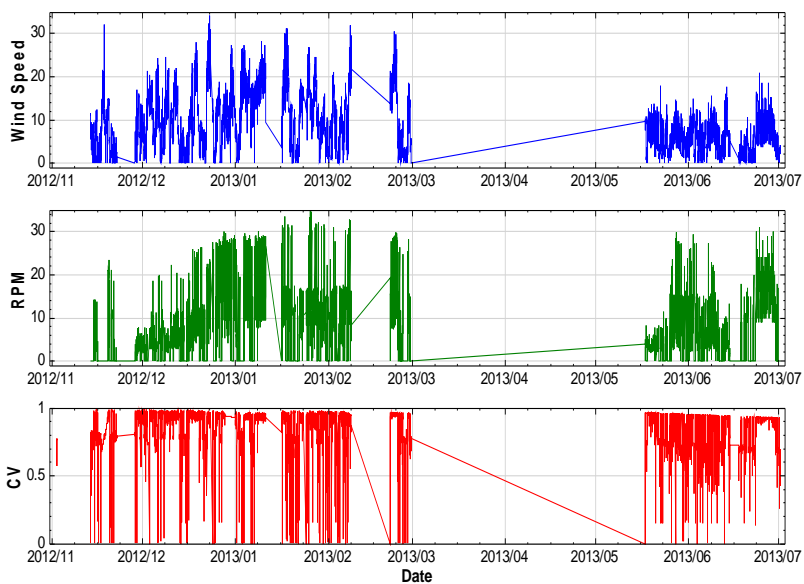

Figure 7. Wind turbine database of original monitoring and assessment data.

2012/11 to 2013/06. Among them, the wind speed (m/sec) and revolution (RPM) are direct recording values, and $\mathrm{CV}$ values are calculated in according with the bearing vibration signal at the corresponding time specified in database. The assessment standard is based on $\boldsymbol{\beta}$ calculated by LR algorithm in accordance with the vibration signal at given normal and abnormal condition and selected CV index.

Upon observing the record variance in Figure 7, the values are not recorded successfully in many intervals. This is because the abnormal connection between field monitoring equipment and database. These abnormal intervals will cause the difficulty of off-line signal processing and analysis. So, the data in successful record intervals are integrated to continuous time series, as 122day records shown in Figure 8.

Because the initial continuous data contain many spikes in Figure 8, these spikes may be generated by the disturbance of sensor or data acquisition equipment, the median filter can be used to remove these spikes effectively to obtain smooth monitoring and assessment data of wind turbine, and the result is shown as Figure 9.

The fine solid line shown at upper part of Figure $\mathbf{1 0}$ is the amplification result of $C V$ ordinate of Figure 9. Upon observing the $C V$ variance trend, it is found that many $C V$ values assessed by LR will be shifted to around 0.8 . The reason for $C V$ shift is that the original bearing vibration signals used for training LR coefficients do not represent normal operation condition of these intervals, including constant speed at different revolution, accelerating and decelerating operation, and stationary condition etc. According to the wind speed and revolution of these intervals, the LR algorithm can complete the representation of the coefficients; then, it can be used to modify previous $C V$ values off-line, and the results are shown as bold dotted line at upper part of Figure $\mathbf{1 0}$ and fine solid line shown at lower part of Figure 10. The $C V$ values 

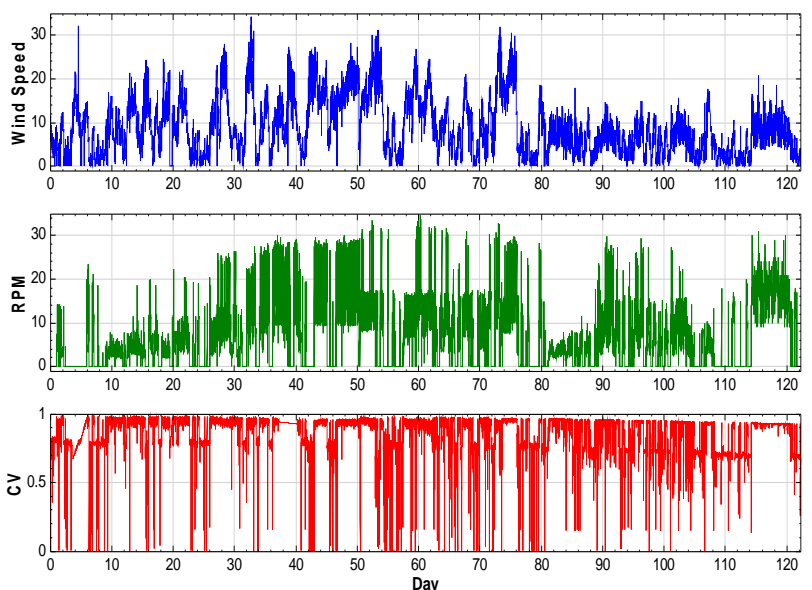

Figure 8. Wind turbine time series of continuous monitoring and assessment data.

will not shift significantly after modification. Upon observing the variance of $C V$, it is composed of gentle degradation trend and high frequency fluctuation. The iterative Gaussian filter proposed in [20] is adopted to smooth the historical records of $C V$ to obtain the medium and highly smooth $C V$ trend shown as bold dotted line and bold solid line at lower part of Figure $\mathbf{1 0}$ for the further prediction step. The iterative Gaussian filter is used as a low-pass filter to extract the non-sinusoidal part of the historical records of $C V$. The cutoff frequencies are selected as 0.2 cycle/day in medium smooth case and as 0.02 cycle/day in highly smooth case, respectively.

\section{Performance Prediction of Wind Turbine Bearing}

\subsection{ARMA Algorithm [21,22]}

The Autoregressive Moving Average (ARMA) algorithm is a system identification model. According to the collected historical performance data of wind turbine bearing, it is able to identify relevant parameters sufficient to represent the performance behavior, and predict the future performance trend in accordance with ARMA model. ARMA model is composed of AR model and MA model, which can be expressed in Equation (4), where $y$ represents the output of system, $x$ represents the input of system, $a$ and $p$ represent the coefficient and order of AR model, and $b$ and $q$ represent the coefficient and order of MA model.

$$
y_{i}=\sum_{j=1}^{p} a_{j} y_{i-j}+\sum_{j=1}^{q} b_{j} x_{i-j}
$$

In Equation (4), it is found that ARMA model is a general equation of linear differential equation, which can be used to represent common linear systems. A general mechanical system can be simplified to the combination of many multi-degree-of-freedom systems. So,
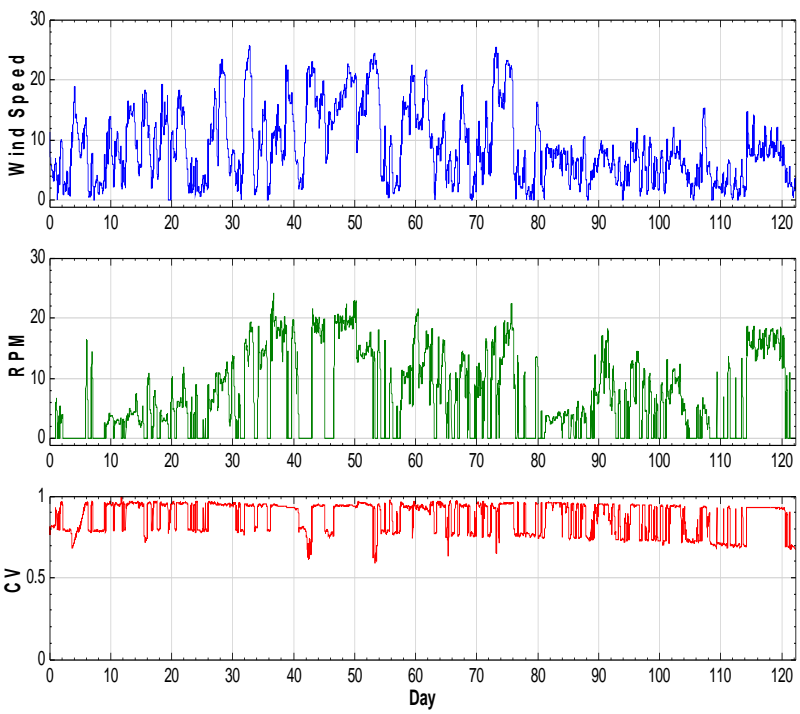

Figure 9. Wind turbine time series of smoothed monitoring and assessment data.
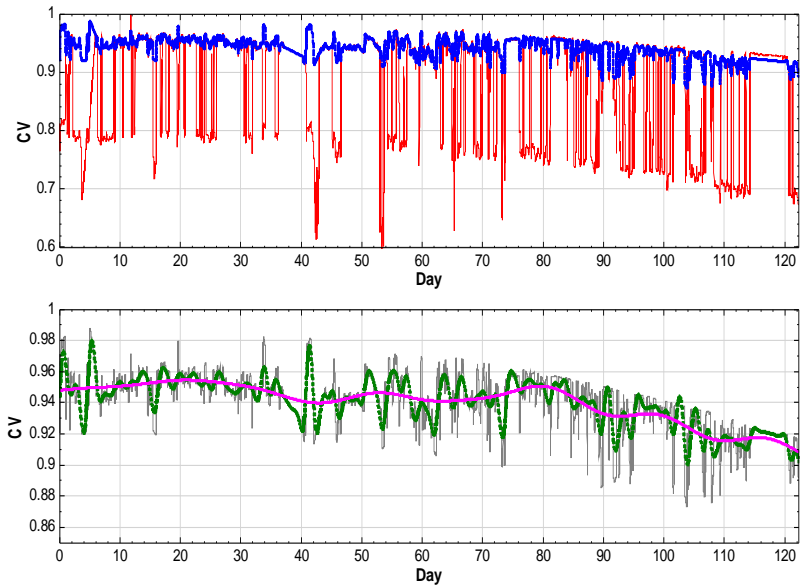

Figure 10. Off-line processing for assessment data of wind turbine bearing.

ARMA model is often used to represent the feature of mechanical system at time domain and frequency domain.

\subsection{Performance Trend Prediction Test of Wind Turbine Bearing}

Figure 11 shows the use of 90-day historical CV data for training ARMA model and future performance trend prediction result of next 32 days. Figure 12 shows the use of 100-day historical CV data for training ARMA model and future performance trend prediction result of next 22 days. The historical CV data are divided into two sections. The first section is used for training and shown as blue solid line, and the second section is used for testing and shown as green dashed line. The performance trend forecasted by ARMA model is shown as red dotted line. Upon observing CV trend of actual assessment, it is 

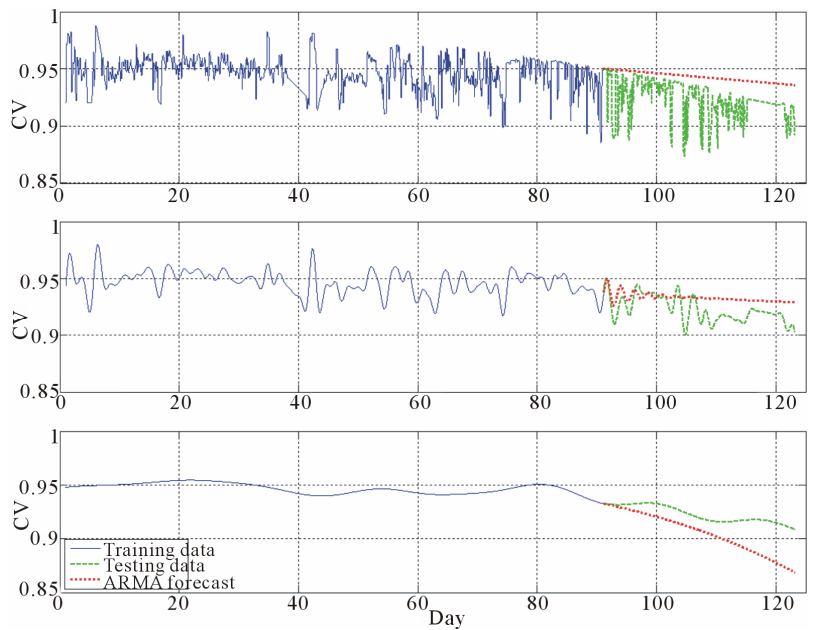

Figure 11. Performance trend prediction result of wind turbine bearing: 90 days of training data.
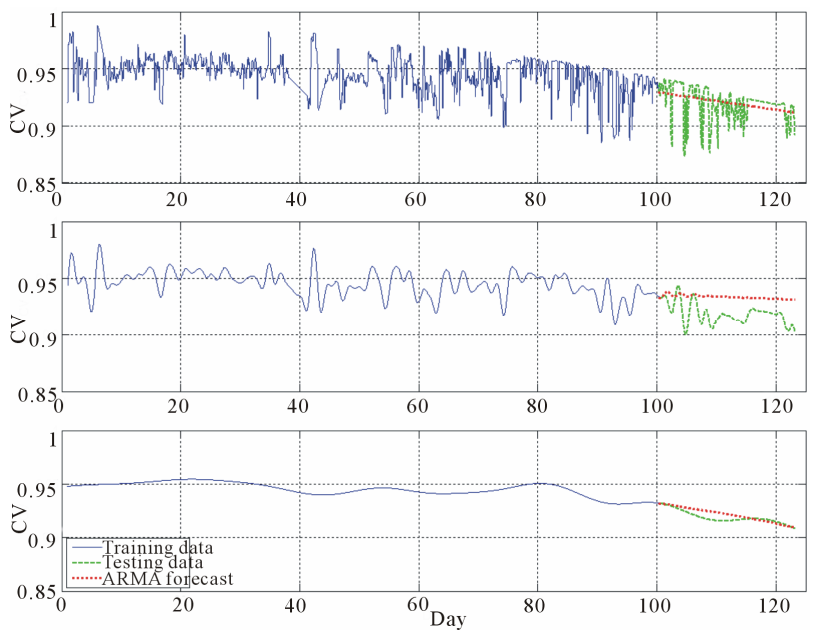

Figure 12. Performance trend prediction result of wind turbine bearing: 100 days of training data.

found that CV has significant degradation trend about 80 days after. So, the prediction deviation is larger by using 90-day historical CV data for training ARMA model. After comparison, the prediction accuracy is higher by using 100-day historical CV data for training ARMA model. In Figure 11, the performance predictions are overestimated by using the original and medium smooth historical CV data, and the prediction is underestimated by using the highly smooth historical CV data. In Figure 12, the performance predictions are correlated well to the testing data by using the original and highly smooth historical CV data, but the prediction is still overestimated by using the medium smooth data.

The historical CV smoothing degree will also influence the prediction result of ARMA model. So, the system developed by this study will re-train the prediction ARMA model regularly and determine the influence of different $C V$ historical data length and smoothing degree on prediction ARMA model. Then, the system uses the rule as the basis of dynamic ARMA parameter adjustment and verifies the reliability of prediction model.

\section{Fault Classification of Wind Turbine Bearing}

\subsection{SVM Algorithm [23-25]}

The Support Vector Machine (SVM) algorithm is a supervised learning classifier derived from the statistical learning theory. Unlike other learning network, the goal of SVM is to minimize structural risk not to minimize empirical risk. So, it is guaranteed as the optimal classifier after learning process. SVM mainly uses binary classification way to classify the samples. As shown in Figure 13, SVM transfers the samples from original space to the feature space for really classifying the samples by a linear hyperplane, and guarantees that two classes of samples have equal distance from the plane. Finally, this hyperplane is transferred back to original space to obtain optimal nonlinear classification curve for two classes of samples. If more than two classes of classification are required, the binary SVM classifier can be expanded to multiple SVM classifiers. Figure 14 shows the structure of Decision Directed Acyclic Graph (DDAG) SVM classifier, which can be used for classifying 4 classes of fault. In Figure 14, $D_{i j}(x)$ represents the binary classifier of $i$ class and $j$ class. According to the directed decision flow to conduct several binary classification, the sample $x$ will finally be able to be classified successfully.

\subsection{Bearing Fault Classification Test}

Figure 15 shows the bearing vibration signal and its shorttime Fourier spectrogram. In the figure, the 6-second

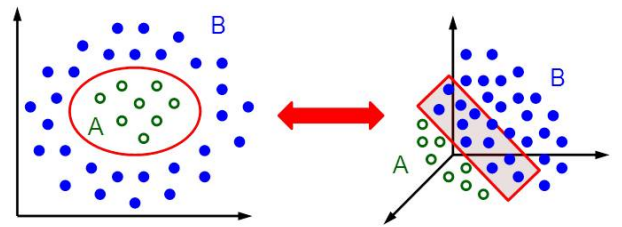

Figure 13. Binary SVM classifier [23].

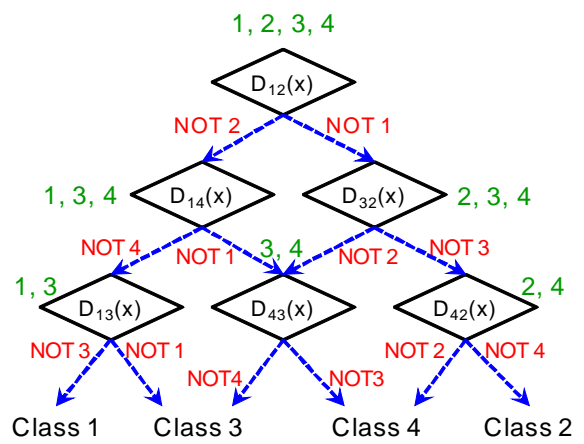

Figure 14. Multiple DDAG SVM classifier [24]. 

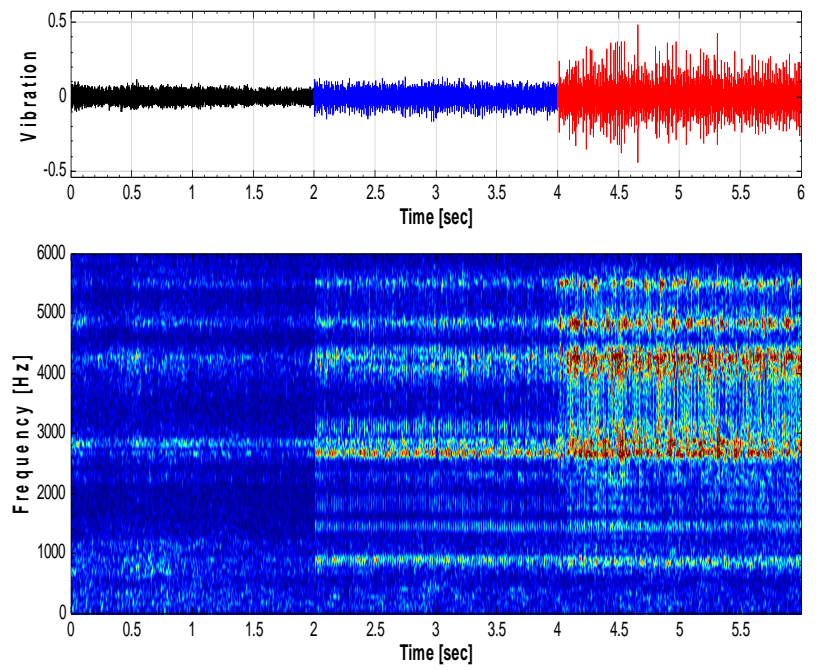

Figure 15. Bearing vibration signal and short-time Fourier analysis.

vibration signal is composed of three 2-second segments, in order to compare the bearing vibration waveform and frequency component between normal and abnormal condition. From the vibration signal, the first segment represents normal bearing, the middle one slightly abnormal bearing, and the last one significant abnormal bearing.

Upon observing the amplitude variance of waveform and spectrogram, it is found when the bearing is abnormal, the amplitude modulation will be occurred, and the modulated frequency will be correlated to bearing characteristic frequency. Figure 16 shows the comparison result of vibration envelope spectrum and characteristic frequencies for bearing vibration signal specified in Figure 15. In the figure, BPFO represents the ballpass frequency at outer race, BPFI represents the ballpass frequency at inner race, BSF represents the ballspin frequency, and FTF represents the fundamental frequency. The calculation is shown in Equations (5)-(8) [26].

$$
\begin{array}{r}
\text { BPFO }=\frac{n}{2}\left(1-\frac{d}{D} \cos \phi\right) f_{r} . \\
\text { BPFI }=\frac{n}{2}\left(1+\frac{d}{D} \cos \phi\right) f_{r} . \\
\text { BSF }=\frac{D}{2 d}\left[1-\left(\frac{d}{D} \cos \phi\right)^{2}\right] f_{r} . \\
\text { FTF }=\frac{n}{2}\left(1-\frac{d}{D} \cos \phi\right) f_{r} .
\end{array}
$$

where $n$ is ball number, $d$ is ball diameter, $D$ is bearing pitch diameter, $\phi$ is contact angle, and $f_{r}$ is rotation frequency of inner race.

Figure 17 shows multiple SVM classification test result of bearing vibration fault features, in which 8 kinds

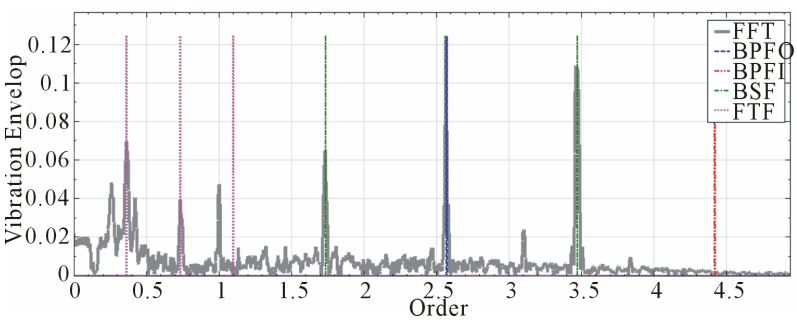

Figure 16. Comparison of vibration envelope spectrum and characteristic frequencies for bearing vibration signal.

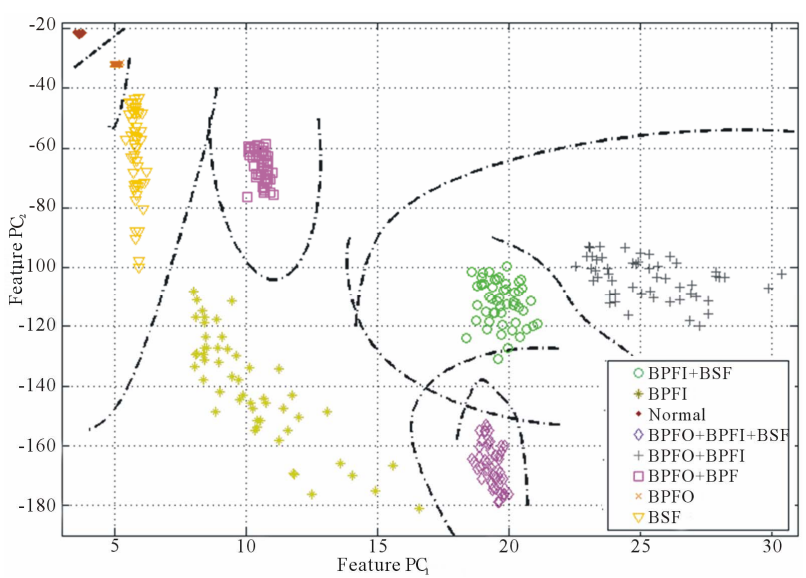

Figure 17. Multiple SVM classification test result of bearing vibration fault features.

of known fault feature are used, including normal condition and other fault combination. In the figure, the abscissa is the first principle component $\mathrm{PC}_{1}$ of signal feature, and the ordinate is the second principle component $\mathrm{PC}_{2}$ of signal feature. The center lines represent the classification curves of fault. The results show that multiple SVM classifiers can distinguish different bearing fault classification effectively. The feature series can be obtained after comparing and analyzing the bearing vibration behaviors at time domain and frequency domain. In order to represent the bearing fault sufficiently, at least 10 kinds of feature are required in general. If the original feature series is used to conduct the classification directly, not only the SVM classification computing efficiency will be poor due to high dimension of feature series, but also the distribution of feature space may be overlapped to cause difficult SVM classification. So, the Principle Component Analysis (PCA) will be matched with SVM classifier to reduce the dimension of feature series, where only the most key components will be used for faster and more accurate classification [16-18].

\section{Conclusion}

The final purpose of this study is to set up the intelligent monitoring system for predictive maintenance of wind turbine, particularly the monitoring, assessment, and diagnosis for key components, such as bearing and gearbox, 
so that the user can predict the best replacement timing of the abnormal component in advance, and grasp the usability and maintenance cost. The condition monitoring and prediction system of wind turbine bearing integrates three functional modules. Firstly, the real-time bearing performance assessment module retrieves current bearing vibration signal from wind turbine database and adopts the LR algorithm to access current $C V$ value. Then, it transfers $C V$ value back to the database. Secondly, when the $C V$ historical data of bearing performance are accumulated to a sufficient number, the ARMA algorithm is adopted to predict the future variation trend of bearing performance in accordance with known $C V$ historical data of bearing performance and assure if the predicted performance is lower than the threshold value. Then, it transfers relevant predicted data back to the database. Thirdly, when the identification for reason of bearing performance reduction is required, the SVM algorithm is adopted to determine the fault classification through retrieving bearing vibration signal at fault interval from wind turbine database. Then, it transfers fault condition data and corresponding records back to the database in order to strengthen the completeness of bearing fault condition database, which not only can modify the coefficient correctness of LR algorithm, but also can be used as the reference for operation parameter adjustment and modification of follow-up design.

\section{Acknowledgements}

The financial support provided by Bureau of Energy (Grant No.102-D0105) is gratefully acknowledged.

\section{REFERENCES}

[1] P. Tavner, "Offshore Wind Turbines: Reliability, Availability and Maintenance,” The Institution of Engineering and Technology, London, 2012.

[2] P. A. Lynn, "Onshore and Offshore Wind Energy: An Introduction,” John Wiley \& Sons Ltd., Chichester, 2012.

[3] R. Billinton, R. Karki and A. K. Verma, "Reliability and Risk Evaluation of Wind Integrated Power Systems," Springer, London, 2013. http://dx.doi.org/10.1007/978-81-322-0987-4

[4] C. R. Farrar and K. Worden, "Structural Health Monitoring: A Machine Learning Perspective," John Wiley \& Sons Ltd., Chichester, 2013.

[5] C. J. Crabtree, "Condition Monitoring Techniques for Wind Turbines,” Ph.D. Thesis, Durham University, Durham, 2011.

[6] F. P. G. Márquez, A. M. Tobias, J. M. P. Pérez and M. Papaelias, "Condition Monitoring of Wind Turbines: Techniques and Methods," Renewable Energy, Vol. 46, 2012, pp. 169-178. http://dx.doi.org/10.1016/j.renene.2012.03.003

[7] K. Tracht, G. T. Goch, P. Schuh, M. Sorg and J. F. Wester- kamp, "Failure Probability Prediction Based on Condition Monitoring Data of Wind Energy Systems for Spare Parts Supply," Manufacturing Technology, Vol. 62, 2013, pp. 127-130.

[8] D. N. P. Murthy and K. A. H. Kobbacy, "Complex System Maintenance Handbook,” Springer, London, 2008.

[9] R. Manzini, A. Regattieri, H. Pham and E. Ferrari, "Maintenance for Industrial Systems," Springer, London, 2010. http://dx.doi.org/10.1007/978-1-84882-575-8

[10] H. Czichos, "Handbook of Technical Diagnostics: Fundamentals and Application to Structures and Systems," Springer, London, 2013.

http://dx.doi.org/10.1007/978-3-642-25850-3

[11] V. Palade, C. D. Bocaniala and L. Jain, "Computational Intelligence in Fault Diagnosis,” Springer, London, 2006. http://dx.doi.org/10.1007/978-1-84628-631-5

[12] V S. Nandi, S. Choi and H. Meshgin-Kelk, "Electric Machines: Modeling, Condition Monitoring, and Fault Diagnosis,” CRC Press, New York, 2012.

[13] D. J. Inman, C. R. Farrar, V. L. Junior and V. S. Junior, "Damage Prognosis: For Aerospace, Civil and Mechanical Systems,” John Wiley \& Sons Ltd., Chichester, 2005. http://dx.doi.org/10.1002/0470869097

[14] B. L. Song and J. Lee, "Framework of Designing an Adaptive and Multi-Regime Prognostics and Health Management for Wind Turbine Reliability and Efficiency Improvement," International Journal of Advanced Computer Science and Applications, Vol. 4, No. 2, 2013, pp. 142-149.

[15] G. Vachtsevanos, F. L. Lewis, M. Roemer, A. Hess and B. $\mathrm{Wu}$, "Intelligent Fault Diagnosis and Prognosis for Engineering Systems,” John Wiley \& Sons Ltd., New Jersey, 2006. http://dx.doi.org/10.1002/9780470117842

[16] M. Kantardzic, "Data Mining: Concepts, Models, Methods, and Algorithms,” 2nd Edition, John Wiley \& Sons Ltd., New Jersey, 2011. http://dx.doi.org/10.1002/9781118029145

[17] P. Xanthopoulos, P. M. Pardalos and T. B. Trafalis, "Robust Data Mining,” Springer, London, 2013. http://dx.doi.org/10.1007/978-1-4419-9878-1

[18] T. Hastie, R. Tibshirani and J. H. Friedman, "The Elements of Statistical Learning: Data Mining, Inference, and Prediction,” 2nd Edition, Springer, London, 2009.

[19] J. Yan, "Degradation Assessment and Fault Modes Classification Using Logistic Regression,” Journal of Manufacturing Science and Engineering, Vol. 127, No. 4, 2005, pp. 912-914. http://dx.doi.org/10.1115/1.1962019

[20] Y. N. Jeng, P. G. Huang and Y. C. Cheng, "Decomposition of One-Dimensional Waveform Using Iterative Gaussian Diffusive Filtering Methods," Proceedings of the Royal Society A, Vol. 464, No. 2095, 2008, pp. 16731695. http://dx.doi.org/10.1098/rspa.2007.0031

[21] P. C. Young, "Recursive Estimation and Time-Series Analysis: An Introduction for the Student and Practitioner," 2nd Edition, Springer, London, 2011. http://dx.doi.org/10.1007/978-3-642-21981-8

[22] M. Najim, "Modeling, Estimation and Optimal Filtration in Signal Processing,” John Wiley \& Sons Ltd., New Jer- 
sey, 2010.

[23] L. Wang, "Support Vector Machines: Theory and Applications,” Springer, New York, 2010.

[24] S. Abe, "Support Vector Machines for Pattern Classification,” 2nd Edition, Springer, New York, 2010. http://dx.doi.org/10.1007/978-1-84996-098-4
[25] C. Campbell and Y. Ying, "Learning with Support Vector Machines,” Morgan \& Claypool Publishers, 2011.

[26] C. Scheffer and P. Girdhar, "Practical Machinery Vibration Analysis and Predictive Maintenance," Elsevier, 2004. 